\title{
The Relation Between the Internuclear Distances and Force Constants of Molecules and Its Application to Polyatomic Molecules
}

\author{
Richard M. Badger, Gates Chemical Laboratory, California Institute of Technology
}

(Received August 10, 1935)

\begin{abstract}
The relation between the internuclear distances and force constants found for diatomic molecules is discussed, and is shown to carry over to polyatomic molecules. It is shown that internuclear distances of polyatomic molecules can be predicted from vibrational data alone, with considerable accuracy.
\end{abstract}

$\mathrm{O}^{\mathrm{N}}$ NE would expect, on theoretical grounds, that there should be a relation between the internuclear distances and the constants of the potential function of a molecule. Though the complete relation must necessarily be very complex the writer observed several years ago, in the case of diatomic molecules, that the equilibrium internuclear distance, $r_{c}$, can be expressed with fair accuracy as a function only of $d^{2} V / d r^{2}$, at the equilibrium separation, and of the positions in the periodic system of the atoms composing the molecules. The fact that such a simple relation can be found is apparently due to the fact that the several factors on which $r$. depends do not vary in an arbitrary and independent fashion through a set of molecules, but change in a more or less parallel manner.

It is to some extent a matter of taste as to how one may best express the relation between $r_{r}$ and $\left(d^{2} V / d r^{2}\right)_{r_{e}}$ or $k_{\rho}$, which may be called the "bond force constant," as the relation is not perfectly exact. The writer believes that an equation which he proposed some time ago, ${ }^{1}$ and which, with a slight modification described below, may be written in the following manner:

$$
r_{e}=\left(C_{i j /} / k_{c}\right)^{\frac{1}{3}}+d_{i j}, \quad \text { Relation I }
$$

is of the most satisfactory form, since with the proper choice of constants it fits very accurately the various electronic states of a given molecule, with the exception of a few abnormal states.

Two other rules have been more recently proposed, ${ }^{2,3}$ which for comparison with Relation I may be rewritten as follows:

1 R. M. Badyer, J. Chem, Phys. 2, 128 (1934).

2 C. H. Douglas Clark, Phil. May. 18, 459 (1934).

3 H. S. Allen and A. K. Longair, Nature 135, 764 (193.5).

$$
\begin{aligned}
& r_{i}{ }^{2}=C_{i j}{ }^{\prime}\left(\mu / n k_{e}\right)^{3} . \quad \text { Clark rule. } \\
& r_{c}{ }^{2}=C_{i j}{ }^{\prime \prime} / k_{e}{ }^{3} . \text { Allen, Longair rule. }
\end{aligned}
$$

These rules fit quite well within a certain range but they do not appear to be of as satisfactory a form as Relation I for fitting the various excited states of a given molecule, and have not the latitude of the two constant formulas necessary for getting a good fit for the heavy molecules.

All the relations mentioned are at best a compromise, and attempt to fit a considerable amount of data with the smallest possible number of arbitrary constants. As was mentioned, Relation I has a suitable form for fitting most states of a given molecule with a single pair of constants, but to get an accurate fit $C$ and $d$ must be given values considerably different from those which give the best average fit for a group of molecules. It might seem reasonable that $C$ and $d$ could be expressed as functions of the atomic numbers of the atoms composing the molecule, which might have an effect similar to that of the parameter $n$ in the Clark equation. This might be possible for the symmetrical molecules for which the constants appear to vary in a systematic manner, but for unsymmetrical molecules they behave in an erratic fashion. This fact, together with the occurrence of some abnormal states which deviate from any simple rule, indicates that any more refined rule must take into account the type of binding present in each state.

The writer has had considerable success in adding to his relation a correction in $\mu \omega_{r} x_{c}$ but so far has not found a rule which makes more accurate predictions than Relation $I$ and gives a unique answer unless the type of bond in question is known, which is often not the case. 
However, Relation I can be improved somewhat, still keeping $C$ and $d$ constant through a group of molecules, if $C$ is allowed to vary with $d$ from group to group, instead of taking a universal value as was previously done. This necessitates taking slightly different values for $d_{i j}$, as given in Table I.

TABLE I.

\begin{tabular}{|c|c|c|c|c|}
\hline $\begin{array}{l}\text { TYPE OF } \\
\text { ATOM } i \\
\text { AN ELE. } \\
\text { MENT IN } \\
\text { ROW }\end{array}$ & $\begin{array}{l}\text { OLECULE } \\
\text { ATOM } j \\
\text { AN ELE- } \\
\text { MENT IN } \\
\text { ROW }\end{array}$ & Example & $\left(C_{i j}\right)^{\prime}$ & $d_{i j}(A)$ \\
\hline 1 & 1 & $\mathrm{NO}, \mathrm{O}_{2}, \mathrm{NO}$ & 0.571 & 0.68 \\
\hline 1 & 2 & $\mathrm{SO}, \mathrm{PN}, \mathrm{CS}$ & 0.535 & 0.94 \\
\hline 1 & 3 & TiO & $(0.500)$ & $(1.06)$ \\
\hline 1 & 4 & $\mathrm{SnO}$ & $(0.490)$ & $(1.18)$ \\
\hline 1 & 5 & $\mathrm{PbO}$ & $(0.490)$ & $(1.26)$ \\
\hline 2 & 2 & $\mathrm{Cl}_{2}, \mathrm{~S}_{2}, \mathrm{P}_{2}$ & 0.490 & 1.25 \\
\hline 2 & 4 & $\mathrm{ICl}$ & $(0.505)$ & $(1.48)$ \\
\hline 3 & 3 & $\mathrm{Br}_{2}, \mathrm{~K}_{2}$ & 0.505 & 1.48 \\
\hline 4 & 4 & $I_{2}$ & $(0.49)$ & $(1.76)$ \\
\hline
\end{tabular}

Parentheses indicate that constants were determined from data on only one molecule except in the case of $\mathrm{S}_{n} \mathrm{O}$ where the values were estimated with the assistance of the althor's Relation II. ${ }^{4}$ The dimensions and units of $C$ are such as to give $r_{e}$ in Angstrom units when $k_{e}$ is given in megadynes per certimeter.

\section{Application to Polyatomic Molecules}

The relation between $k_{e}$ and $r_{e}$ which has been found to exist for diatomic molecules has proved to be useful in a number of ways. It is the purpose of this paper to enquire whether this relation carries over, in general, to the polyatomic molecules. The number of cases in which all, or even any of the internuclear distances in poly. atomic molecules can be exactly evaluated from the analysis of the rotational structure of their bands is definitely limited, for obvious reasons. On the other hand, a great deal of information about the vibrational structure of the spectra of such molecules has already accumulated and if it can be made available for determining internuclear distances several interesting possibilities will be opened up.

These vibrational data have already been used, in the cases of several typical molecules, in evaluating the constants of simple potential functions. With one or two exceptions one has been content with potential functions containing not higher than quadratic terms, but it is the coefficients of certain of these quadratic terms

${ }^{4}$ R. M. Badger, Phys. Rev, 48, 284 (1935). which interest us at present, as we wish to study their relation to the internuclear distances.

In making an examination of this sort one is confronted with several difficulties. In the first place there are few cases where one knows exact internuclear distances and at the same time has complete vibrational data available. To make even the ordinary normal coordinate treatment of a molecule, to obtain the second derivatives of the potential energy with respect to the various coordinates, one should employ the normal frequencies of vibration for infinitesimal amplitude. These are obtained through an extrapolation from the observed frequencies which it is usually not possible to make on account of the incompleteness of the data. However, it seems improbable that the approximation introduced in making use directly of the observed frequencies of the fundamental bands and Raman lines will lead to serious error in the important force constants except in the case of light molecules, particularly the hydrogen-containing molecules.

But now we are faced with another difficulty in regard to the form of potential function which we shall attempt to apply to molecules. It would of course be desirable to start with the most general function containing all possible quadratic terms. But usually, even for rather simple molecules, we should find that we have insufficient data to evaluate all the constants. Furthermore, even the most general function can usually be written in several different forms which are equivalent, but which suggest different physical interpretations of the molecule.

Now in the study of many properties of molecules it has been found that they may be regarded as the sum of several individual parts which to a good approximation may be regarded as independent. So also it is convenient to regard the potential energy as made up of a sum of various rather independent parts, and one criterion for selecting a suitable form of function would be that it should be suggestive of the concept of a molecule which has been developed from other experimental information. If this concept is sufficiently well developed we should expect it to be a good guide in deciding which of the constants of the potential function are small and may be ignored and which we shall 
attempt to determine from the limited data available.

These expectations appear to be justified. For a number of different types of molecules so far investigated there appears to be one general method of setting up the potential function which is preferable to other methods. The potential energy is regarded as made up of three main parts, as far as concerns the quadratic terms. Part $\mathrm{I}$, and the most important, is regarded as dependent only on the distances between the atoms which are directly bonded to each other. For small displacements from the equilibrium position the change in energy is given by:

$$
2 V_{r}=\Sigma k_{e_{i}}\left(\Delta r_{i}\right)^{2}
$$

For larger extensions or compressions of a bond there is some evidence that a Morse function may be applied.

In the case of certain molecules which we shall discuss first, where the bonds are not under appreciable tension, we may designate the coefficients of the terms of Part I as "bond force constants," represented again by the symbol $k_{e}$, rather than $k_{f}$, and may compare them directly with these constants for diatomic molecules.

Part II of the energy is dependent on the angles between chemical bonds, and Part III, which must be added to complete the function so that it will fit the vibrational data, contains terms which may be interpreted as arising from interactions between atoms not directly bonded to each other. When the potential function is written as described these interaction terms are usually rather small and sometimes may be ignored. However, it is occasionally important how they are written, owing to the effect on the magnitude of the $k_{e}$ 's. From the data so far available it appears that the interactions mentioned result mainly from the repulsions of nonbonded atoms and the interaction terms should be written so as to suggest this fact.

Now in writing the potential energy as described we shall frequently introduce a redundancy of coordinates, in which case we expect linear terms also to appear in the complete expression. These will not concern us immediately though indirectly they are of importance.
The fact which is now of interest is that in a potential function written as above described the $k_{e}$ 's do appear to have the same relation to the $r_{e}$ 's as was found for diatomic molecules. This fact will now be illustrated by a number of cases.

\section{Molecules with Bonds Under Little TENSION}

In the first cases considered as exact a test as possible will be made and instead of employing Relation I with the constants given in Table I, a plot of $\left(1 / k_{e}\right)^{t}$ against $r_{e}$ will be given for the atom pairs: $\mathrm{O}-\mathrm{H}, \mathrm{C}-\mathrm{O}, \mathrm{C}-\mathrm{S}$, and $\mathrm{S}-\mathrm{O}$, both when found as a diatomic molecule ${ }^{5}$ and when constituting part of a polyatomic molecule. It will be seen that for a given atom pair all points fall very close to a straight line.

The most satisfactory tests are for $\rho-\mathbf{H}$ and $\mathrm{C}-\mathrm{O}$, since for both $\mathrm{H}_{2} \mathrm{O}$ and $\mathrm{CO}_{2}$ rather complete vibrational analyses have been made, ${ }^{6}$ and internuclear distances are accurately known. ${ }^{7}$ In the case of $\mathrm{H}_{2} \mathrm{O}$ the interaction terms are small and the value of $k_{e}\left(\mathrm{OH}\right.$ in $\left.\mathrm{H}_{2} \mathrm{O}\right)$ will not be materially affected by how they are introduced. The fit of the $\mathrm{H}_{2} \mathrm{O}$ point with those for the $\mathrm{O}-\mathrm{H}$ molecule will be seen in Fig. 1 to be practically perfect.

In the case of $\mathrm{CO}_{2}$ or $\mathrm{CS}_{2}$ we might write the potential function in the two equivalent forms:

$$
\begin{aligned}
& 2 V=k_{e}\left(\Delta r_{1}^{2}+\Delta r_{2}^{2}\right)+k_{12}\left(\Delta r_{1}+\Delta r_{2}\right)^{2}+\cdots, \\
& 2 V=k_{e}^{\prime}\left(\Delta r_{1}^{2}+\Delta r_{2}^{2}\right)+2 k_{12}\left(\Delta r_{1} \times \Delta r_{2}\right)+\cdots .
\end{aligned}
$$

Now $k_{e}$ and $k_{e}^{\prime}$ differ by $k_{12}$, which is appreciable both for $\mathrm{CO}_{2}$ and $\mathrm{CS}_{2}$, but there seems to be no doubt but that the former is the one which we should compare with the data on $\mathrm{CO}$ and $\mathrm{CS}$, respectively, since the vibrational analysis of $\mathrm{CO}_{2}$ by Adel and Dennison shows that the interaction term arises mainly from a repulsion of the oxygen atoms, indicating that the former

\footnotetext{
${ }^{5}$ The data for diatomic molecules have been taken from the report of Jevons (Physical Society) except where more recent work is available.

( $\left(\mathrm{H}_{2} \mathrm{O}\right)$ L. G. Bonner, Phys. Rev. 46, 458 (1935): $\left(\mathrm{CO}_{2}\right)$ Adel and Dennison, Phys. Rev, 44, 99 (1933).

${ }_{7}\left(\mathrm{H}_{2} \mathrm{O}\right)$ Freudenberg and Mecke, Zeits. f. Physik 81, 465 (1933); $\left(\mathrm{CO}_{2}\right)$ Barker and Adel, Phys. Rev. 44, 185 (1933).
} 


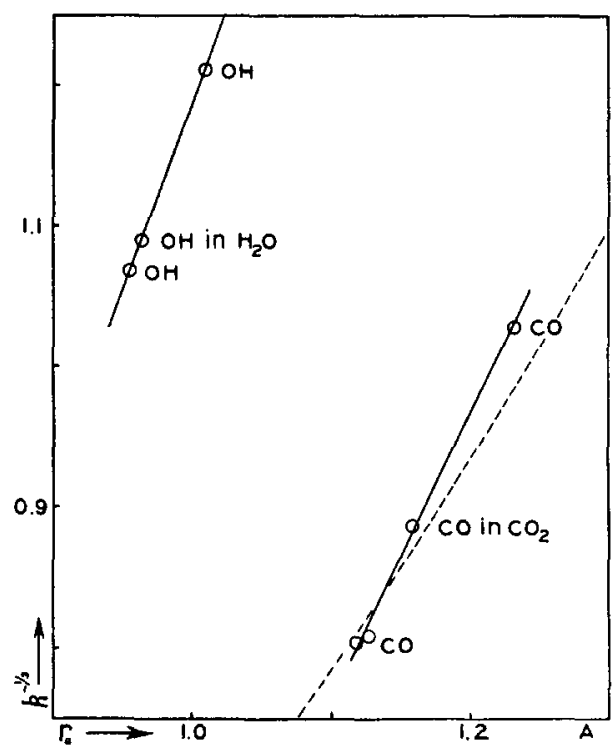

FIG. 1. A plot of $\left(1 / k_{e}\right)^{\prime}$ against $r_{e}$ for the atom pairs: $\mathrm{O}-\mathrm{H}$ and $\mathrm{C}-\mathrm{O}$, when found as diatomic molecules or in the $\mathrm{H}_{2} \mathrm{O}$ and $\mathrm{CO}_{2}$ molecules, respectively. The units of $k_{\varepsilon}$ are megadynes per $\mathrm{cm}$. The dotted line shows the locus of points for the $\mathrm{CO}$ molecule predicted by the $\mathrm{C}$. $\mathrm{H}$. Douglas Clark rule.

expression of the potential function is to be preferred. For $\mathrm{CO}_{2}$ the fit with the data on the diatomic molecule is again seen to be very good if $k_{e}$ is used, as in Fig. 1, but with $k_{e}{ }^{\prime}$ the point deviates about $0.015 \mathrm{~A}$ from the line as drawn. In the case of $\mathrm{CS}_{2}$ and also $\mathrm{SO}_{2}$ we must be content with a less accurate test, as we are obliged to use internuclear distances determined by electron diffraction methods, ${ }^{8}$ in which the error may be as large as $0.02 \mathrm{~A}$, and in the normal coordinate treatment the observed fundamentals have been employed rather than the frequencies for infinitesimal amplitude. In $\mathrm{SO}_{2}{ }^{9}$ the interaction between the oxygen atoms has been ig. nored, which may lead to some error. In spite of these approximations the fit for $\mathrm{CS}_{2}$ and $\mathrm{SO}_{2}$ is still quite satisfactory, as seen in Fig. 2, as the deviation from the straight line is of the order

\footnotetext{
${ }^{8}$ Private communication from Dr. L. O. Brockway.

${ }^{9}$ For the force constants of $\mathrm{SO}_{2}$ the author is indebted to Dr. Paul C. Cross who has made a normal coordinate treatment of this molecule.
}

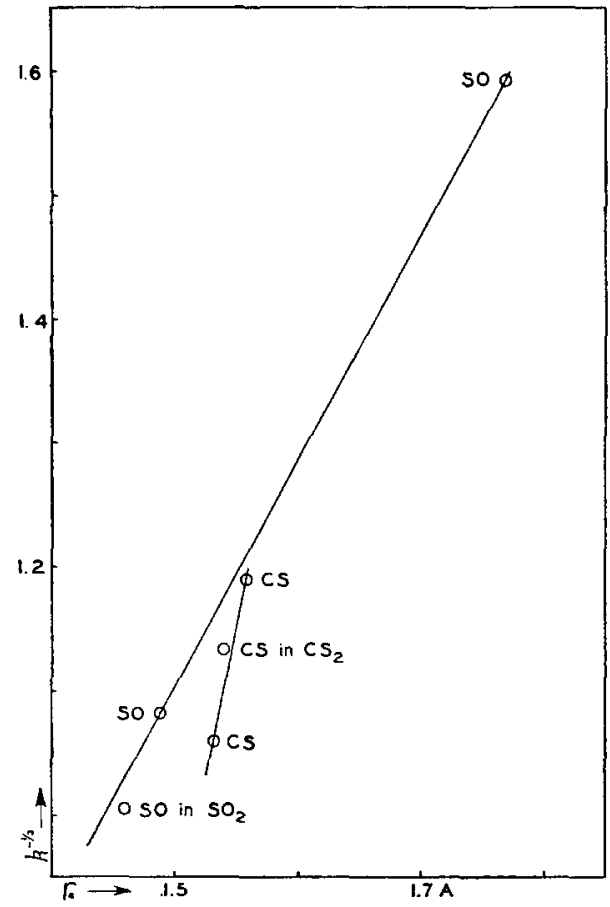

FIG. 2. A plot of $\left(1 / k_{e}\right)$ against $r_{e}$ for the atom pairs $\mathrm{C}-\mathrm{S}$ and $\mathrm{S}-\mathrm{O}$.

of the uncertainty of the measurement of the distance.

\section{Molecules with Bonds Under Tension}

We shall now consider some molecules of a somewhat different type, those with the general formulas: $M X_{4}$ and $M X_{6}$, for example $\mathrm{CCl}_{4}$ and $\mathrm{SF}_{6}$, in which one central atom is surrounded by several relatively large halogen atoms. A satisfactory treatment of these molecules by normal coordinate methods has only recently been made ${ }^{10.11}$ and has shown that the halogen atoms appear to be compressed considerably so that the mutual repulsions result in a stretching of the bonds between the halogen atoms and central atom.

\footnotetext{
${ }^{10}$ Jenny E. Rosenthal, Phys. Rev. 46, 730 (1934).

11 Sachse and Bartholomé, Zeits. f. physik. Chemie B28, 257 (1935).
} 
The vibrational analyses have yielded values for both $d^{2} V_{r} / d r^{2}$ and $d V_{r} / d r$ for the molecules in the equilibrium position, ${ }^{12}$ at which we may call the distance from central to outer atom $r_{t}$. If we assume that a Morse function applies to $V_{r}$ we can calculate the difference between $r_{\varepsilon}$ and $r_{e}$, the distance at which $d V_{r} / d r=0$, or in other words the amount of stretching of the bond. To do this, however, we must assume some value for the constant $a$, appearing in the exponent of the Morse function. The author has found that for a single bond the $a$ may be sufficiently well estimated by the relation :*

$$
a=1.15 /\left(r_{e}-d_{i j}\right) .
$$

Values of $r_{\mathbf{c}}-r_{e}$ calculated in this manner for several molecules appear in Table II.

TABLE II.

\begin{tabular}{|c|c|c|c|c|c|}
\hline Molecule & $\begin{array}{c}k_{\epsilon} \\
\text { (megadynes } \\
\mathrm{cm})\end{array}$ & $\begin{array}{l}\left(d V_{r} / d r\right)_{r_{e}} \\
\text { (megadynes } \\
\left.\times 10^{8}\right)\end{array}$ & $r-r_{e}(A)$ & $r$ (calc.) & $r_{\epsilon}$ (obs.) \\
\hline $\mathrm{CCl}_{4}$ & 0.178 & 0.066 & 0.19 & 1.76 & 1.76 \\
\hline $\mathrm{SiCl}_{4}$ & 0.255 & 0.0464 & 0.12 & 1.98 & 2.02 \\
\hline $\mathrm{SnCl}_{4}$ & 0.227 & 0.0343 & 0.11 & 2.27 & 2.29 \\
\hline $\mathrm{SF}_{6}$ & 0.344 & 0.196 & 0.19 & 1.56 & 1.58 \\
\hline $\mathrm{SeF}_{8}$ & 0.368 & 0.141 & 0.17 & 1.67 & 1.70 \\
\hline $\mathrm{TeF}_{6}$ & 0.395 & 0.112 & 0.15 & 1.80 & 1.84 \\
\hline
\end{tabular}

These results are very interesting since we observe that when one or more of the halogen atoms, say in $\mathrm{CCl}_{4}$, are replaced by the small hydrogen atom, allowing the remainder to separate somewhat and to take up a new and more "comfortable" position, they do not actually shrink in appreciably. The $\mathrm{C}-\mathrm{Cl}$ dis-

${ }^{12}$ For the hexafluorides the constants in columns II of the table of Sachse and Bartholomé have been used.

* Note added in proof: This is an empirical rule which appears to serve fairly well in the comparison of similar molecules. The constant varies from one type of molecule to another and in the case of the fluorides 1.05 was used in the calculations though a still smaller value would have given better agreement with experiment. tance is, within experimental error, the same in $\mathrm{CCl}_{4}$ and $\mathrm{CH}_{3} \mathrm{Cl}$. It appears then that the same Morse function cannot be applied to the $\mathrm{C}-\mathrm{Cl}$ bond in the two cases, and the constancy of the $\mathrm{C}-\mathrm{Cl}$ distance seems to be a somewhat accidental affair arising from certain compensations.

Now we shall proceed to apply Relation I to molecules of the type just discussed. Evidently we cannot expect to do this directly since the experimental data give us values of $r_{\epsilon}$ and $k_{\epsilon}$ or $d^{2} V_{r} / d r^{2}$ at $r_{e}$, and the expression relates $r_{e}$ and $k_{e}$. However, we may apply the Morse function again to correct $k_{\epsilon}$ to $k_{t}$, then calculate $r_{e}$ by Relation I, and correct it back to $r_{\epsilon}$.

In this way, by using the constants in Table I, $r_{c}$ has been calculated for several molecules and in Table II is compared with the experimental value obtained from electron diffraction measurements. ${ }^{13}$ In only two cases does the deviation greatly exceed the possible error in the electron diffraction measurements. The error in the calculated value seems to increase with the weight of the central atom which suggests that the method of calculation might be further improved.

\section{Conclusion}

The above tests exhaust the suitable data at present available, but for the types of molecule discussed they do appear to justify the division of the potential energy into the parts previously mentioned and show that the relation between $\left(d^{2} V_{r} / d r^{2}\right)_{r_{e}}$ and $r_{e}$ found for diatomic molecules persists in the polyatomic molecules. This fact should be of considerable use since it provides a method of calculating internuclear distances in these molecules from vibrational data alone, with sufficient accuracy for many purposes.

\footnotetext{
${ }^{13}$ Pauling and Brockway, J. Chem. Phys. 2, 867 (1934); Brockway and Wall, J. Am. Chem. Soc. 56, 2373 (1934); Pauling and Brockway, Proc. Nat. Acad. Sci. 19, 68 (1933).
} 